\title{
¿Por qué las corrientes sociológicas soslayan la investigación biográfica?
}

\author{
Why did sociological currents ignore biographical research?
}

\author{
María Eugenia Cardenal de la Nuez y Francesc J. Hernàndez
}

\section{Resumen}

El artículo muestra cómo las corrientes sociológicas más importantes, la corriente comprensiva y la corriente crítica, dejaron de lado la investigación biográfica. Aunque la hermenéutica de Schleiermacher permitía la investigación de la generalidad en la particularidad, fue la introducción del «grado de evidencia» por parte de Weber el que orientó la corriente comprensiva hacia el funcionalismo estructuralista. Los estudios sobre el campesino polaco o los parados de Marienthal apuntaban una superación de esa restricción, que tardó décadas en producirse. En el segundo caso, la corriente crítica, la tesis de la determinación de la conciencia soslayó la metodología biográfica, aunque tal afirmación ni se encuentra en Marx ni se corresponde con su proyecto teórico. Por último, se analiza la excepción de la biografía de Offenbach de Kracauer.

\section{Palabras clave}

Investigación biográfica, Schleiermacher, tipos ideales, Escuela de Chicago, Marx, Kracauer.

\section{Abstract}

The article shows how the most important sociological currents, the comprehensive current and the critical current, put aside biographical research. Although Schleiermacher's hermeneutics allowed for the investigation of generality in particularity, it was the introduction of the «degree of evidence» on the part of Weber that guided the comprehensive current toward structuralist functionalism. Studies on the Polish peasant or the Marienthal unemployed pointed to an overcoming of that restriction, which took decades to occur. In the second case, the critical current, the thesis of the determination of consciousness, ignored the biographical methodology, although such a statement is neither found in Marx nor corresponds to its theoretical project. Finally, the exception of the Offenbach biography of Kracauer is analyzed.

\section{Keywords}

Biographical research, Schleiermacher, ideal types, Chicago School, Marx, Kracauer. 


\section{Introducción}

En el seno de la sociología en general y de la sociología de la educación en particular, la investigación biográfica no ha tenido en el desarrollo que han gozado otras metodologías. Se podría decir que hasta las últimas décadas del siglo xx y de la mano de corrientes como la denominada Segunda Escuela de Chicago, la sociología no desarrolló estudios biográficos en general, ni referidos a la educación en particular. La razón de esta ausencia se debe a que tanto en la corriente comprensiva, como en la corriente crítica de la sociología, las predominantes en la segunda mitad del siglo XIX y el siglo xx, se aceptaron, con una cierta ingenuidad, premisas teóricas que, salvo excepciones, cerraron el paso a la indagación biográfica. Este artículo se dedica a exponer las razones que se invocaron para ejecutar aquella restricción metodológica. Se trata pues de un artículo teórico, que selecciona y comenta el conjunto de textos donde se justificó el soslayamiento de lo biográfico..

\section{La corriente comprensiva}

\section{1. La hermenéutica}

Cuando Max Weber, en 1904, se hizo cargo, junto con Werner Sombart y Edgar Jaffé, de la coedición de la revista de investigación social Archiv für Sozialwissenschaft und Sożialpolitik [Archivo para la ciencia social y la política social] efectuó una declaración programática (la nota introductoria al primer número de la nueva serie) y redactó un artículo metodológico crucial: «La “objetividad” del conocimiento científico-social y político-social»). Weber explicó en estos textos que la revista, ampliando su círculo de intereses anteriores...

«tiene que ver en el conocimiento histórico y teórico del significado cultural general del desarrollo capitalista aquel problema a cuyo servicio se encuentra, por lo que tiene que mantenerse en estrecho contacto con las disciplinas vecinas: la teoría general del Estado, la filosofía del Derecho, la Ética social, las investigaciones sociopsicológicas, así como las recogidas generalmente bajo el nombre de sociología». (cit. Weber, Marianne 1995: 433-434).

Como puede verse, hay una cierta confusión terminológica, que tenemos que dejar de lado para ir a lo fundamental de nuestro tema. Para Weber hay, por una parte, un conjunto de «investigaciones sociopsicológicas», así como otras «recogidas generalmente bajo el nombre de sociología» (como serían, p. ej., las teorías de Herber Spencer), y, por otra parte, otro conocimiento más propio, «histórico y teórico», que se encuentra al servicio del problema que es preciso resolver. Resulta muy relevante que en el artículo metodológico mencionado, Weber comienza explicando que:

«Aquel que conozca los trabajos de los lógicos modernos (solo baré mención de Windelband, Simmel

y, para nuestros fines, en especial, de H. Rickert), advertirá pronto que aqui lo esencial se relaciona con ellos». (Weber, Max 1973: 39).

Se aceptaba así una orientación hermenéutica que hundía sus raíces en las obras escritos de Friedrich Ernst Daniel Schleiermacher y ya estaba presente en Sobre la religión (1799) o los Monólogos (cf Poca 1991). Según la primera obra, religión no pretende, como la metafísica, determinar y explicar (erklären) el universo según su naturaleza (y en este Schleiermacher se enfrentó a una posición compartida por muchas filosofías, desde la escolástica hasta Hegel o Schelling); tampoco alberga la pretensión, propia de Kant o Fichte, de perfeccionar el mundo y consumarlo, desde la fuerza y el arbitrio divino del ser humano que constituiría la moral. Frente a estas dos posiciones, Schleiermacher afirma pro- 
gramáticamente de la religión: «Su esencia no es pensamiento ni acción, sino intuición y sentimiento» (Schleiermacher 1985: 35). Escribe más en concreto:

«La religión vive toda su vida [...] en la naturaleza, pero en la naturaleza infinita del conjunto, del Uno y Todo; lo que en esta última es válido para todo ser individualy, de este modo, para todo ser bumano, y alli donde todo, y también el ser humano, puede avanzary permanecer en esta agitación eterna de formas y esencias particulares, la religión quiere, en una silenciosa devoción, intuir y barruntar en lo individual》. (Schleiermacher 1985: 35-36).

Aparentemente, el texto de Schleiermacher tiene poco que ver con el debate sobre las «ciencias del espíritu». Sin embargo, la intuición a la que apela Schleiermacher se irá perfilando como una vía cognoscitiva distinta de la «explicación» y que permitiría concebir lo universal en lo particular, como podemos leer en diversos fragmentos (Schleiermacher 1985: 38-39, 44, 59 y 88-89).

Los Monólogos son textos de una retórica vehemente, que siguen los diálogos de Platón en lo que suponen de clarificación paulatina de una cuestión, aunque en este caso de manera monológica. Algunos pasajes vuelven sobre la vinculación con el Uno y Todo mediante una especie de autognosis, de una vuelta al «conócete a ti mismo»:

«Sobre mi mismo be de volver la mirada, no solo para dejar transcurrir cada momento como una parte del tiempo, sino para aprehenderlo como elemento de la eternidad, y transformarlo en una vida superior y más libre.

Solo hay libertad e infinitud para aquel que sabe qué es el mundo y qué el ser humano. [...] Para mí el espiritu es lo primero y lo único: puesto que lo que reconozco como mundo es su obra más bella, su espejo creado por él mismo. [...] Lo que es digno de ser denominado mundo es solo la eterna comunidad de los espiritus, su influencia mutua, su formarse reciproco, la elevada armonía de la libertads. (Schleiermacher 1868: 31).

Así pues, comprendemos no apelando a datos externos, determinando y explicando, sino volviendo la mirada sobre nosotros mismos, partiendo de la comunidad de espíritus que constituimos los seres humanos; y de este modo, la autognosis proporciona universalidad a nuestro conocimiento.

\subsection{Weber}

En los textos de Economía y sociedad, Weber (1980: 1) asume explícitamente toda la tradición mencionada:

«Sociología (en el sentido que se entiende aqui esta palabra usada de maneras muy diversas) debe significar: una ciencia que pretende comprender (verstehen) de manera interpretativa la acción social y, con ello, explicar (erklären) causalmente su curso y sus efectos».

Como puede verse en el texto, la dualidad, ya apuntada por Schleiermacher, entre explicar y comprender, se ha resuelto a favor de la preponderancia de verstehen. Y prosigue Weber:

"Con ello, debe denominarse «acción» a una conducta bumana (resulta indiferente si es un hacer externo o interno, un omitir o un permitir) siempre y cuando las personas actuantes vinculen a ella un sentido subjetivo». (loc. cit.). 
La cuestión metodológica relevante es cómo el sociólogo descubre el sentido que los individuos vinculan a su acción. La respuesta de Weber recuerda la distinción kantiana entre númeno y fenómeno, entre cosa en sí y cosa para mí. No se trata de aprehender el sentido «objetivamente "correcto"» o «verdadero», de lo que se ocuparían otras ciencias «dogmáticas» (sic), como la jurisprudencia, la lógica, la ética y la estética, "que pretenden investigar en sus objetos el sentido "correcto" o "válido"》, podríamos decir, el sentido en sí. Sino que se trata, en las ciencias empíricas de la acción, a saber, la sociología y la historia, de aprehender el sentido para la persona actuante o para nosotros. Obsérvese este importante fragmento (loc. cit.) sobre el significado que atribuye a «sentido»:

"Aqui el "sentido" es o bien

a) el sentido efectivo

a) en un caso dado bistóricamente de una persona actuante, o

B) el sentido a modo de promedio y aproximación en una masa dada de casos de las personas actuantes, 0

b) el sentido supuesto subjetivamente en unos tipos puros construidos conceptualmente por el sentido o que las personas actuantes los consideren como tipos».

Así pues, Weber abre la puerta a la comprensión del sentido de la acción de una persona (caso a. $\alpha$ ) frente a procedimientos que tienen que ver con una cantidad de casos o con los tipos ideales (caso a. $\beta$ y b). Esta es la puerta por la que accedemos al estudio sociológico de las biografías. Pero es una puerta que se cierra bien pronto.

En esta consideración de los conceptos sociológicos fundamentales en el capítulo de los «fundamentos metódicos», Weber también añade una precisión que cobrará importancia posteriormente. El sociólogo alemán se pregunta por la «evidencia» de la comprensión. No todas las comprensiones de significado resultan igualmente evidentes. Dentro de esta gradualidad, Weber (1980: 3) puntualiza:

«De esta manera, toda interpretación (Deutung) de una acción orientada racionalmente a fines posee-para la comprensión (Verständnis) de los medios empleados-el grado máximo de evidencia».

Adviértase que Weber no restringe la comprensión a la acción orientada racionalmente a fines, sino que simplemente afirma que en ese caso podemos gozar de mayor evidencia. Como veremos, sus discípulos funcionalistas sí que operaron tal restricción.

\subsection{El campesino polaco y los parados de Marienthal}

En la amplia nota metodológica que sus autores, William I. Thomas y Florian Znaniecki, miembros de la llamada Escuela de Chicago, antepusieron a su voluminosa obra El campesino polaco en Europa y América (Thomas \& Znaniecki 1918-1920, I: 1-86), echan en falta una técnica racional aplicable a la realidad social, que ofrezca los «resultados maravillosos» que se obtienen cuando se aplica a la esfera de la realidad material (ibid., 1, cf. Alheit \& Dausien 2009). La necesidad de esta técnica estriba en que, según los autores, se afrontaban dramáticas transformaciones sociales, a las que Thomas también aludió en discurso presidencial de 1927 ante la Sociedad Americana de Sociología. Se producía entonces una «actual y rápida desestabilización de la sociedad, que está relacionada con el asentamiento urbano de la población, la quiebra de los grupos de parentesco, la circulación de la información, la comercialización de las diversiones, etc.» (cit. Alheit \& Dausien 2009). 
En oposición a un «fenómeno físico», un «fenómeno social» debía ser analizado no solo en su contenido objetivo, sino también a partir de su significado para las personas (Thomas \& Znaniecki 1918-1920, I: 38). Por tanto, las actitudes subjetivas y los valores vigentes generales son constitutivos en idéntica medida para la definición de una situación social (Znaniecki 1969: 108, cit. Alheit \& Dausien). Solo de este modo se puede explicar que individuos en circunstancias semejantes reaccionaran de manera completamente diferente ante determinados fenómenos. Parece plausible que las biografías sociales representan un rico material, en el que está documentada la mediación de «datos» objetivos y subjetivos. Respecto al tema de la biografía, Thomas y Znaniecki afirmaron: «Estamos seguros al decir que los recuerdos personales (life-records), tan completos como sea posible, constituyen el tipo perfecto de material sociológico» (Thomas \& Znaniecki 1918-1920, III: 6). Más en extenso:

\section{"[En las ciencias sociales] deben alcanzarse las experiencias y actitudes humanas reales que constitu- yen la realidad social plena, viva y activa debajo de la organización formal de las instituciones sociales, o detrás de los fenómenos de masas estadísticamente tabulados que, tomados en si mismos, no son más que sintomas de procesos causales desconocidos y pueden servir solo como terreno provisional para bipótesis sociológicas». (ibid. 8).}

Según Alheit y Dausien, puesto que las causas de los procesos objetivos de transformación no fueron reflejadas por los sociólogos de Chicago, resultaba natural captar de manera más precisa la variable «subjetividad», más si cabe cuando los grandes movimientos migratorios de comienzos del siglo xx situaban efectivamente como un problema social central la integración o la exclusión de los individuos y de los grupos. Por eso, auspiciados por la Escuela de Chicago, se realizaron estudios sobre temas relacionados con la adaptación del individuo. Se podría pensar que, detrás de este interés biográfico, también hay una operación de fijación del inadaptado en tanto que «caso», como ya denunció Michel Foucault (1981: 196-197).

Otro ejemplo clásico que combinó métodos cuantitativos y cualitativos fue Los parados de Marienthal. En febrero de 1930 se clausuró una importante fábrica téxtil en Marienthal, en la Baja Austria. El Österreichischen Wirtschaftspsychologischen Forschungsstelle [Centro Austríaco de Investigación de Psicología Económica] financió una investigación sobre los desocupados de Marienthal, probablemente a partir de una sugerencia del líder socialdemócrata austríaco Otto Bauer. La investigación fue dirigida por Paul Felix Lazarsfeld y entre sus responsables estuvieron también Marie Jahoda y Hans Zeisel. En 1931 comenzó el trabajo y dos años después se publicó Los parados de Marienthal. Un ensayo sociográfico sobre los efectos del paro de larga duración en la editorial Hirzel de Leipzig. Como se ha dicho, en esta investigación se combinaron métodos cuantitativos y cualitativos (entre ellos: observación estructurada, observación participante, investigación-acción, informes de expertos, material proyectivo, test psicológicos y recuerdos escritos). De todos modos, se otorgó prioridad a los cuantitativos:

"Hemos intentado minimizar los elementos subjetivos inherentes a cualquier observación, rechazando las observaciones no confirmadas por los datos cuantificados». (cit. Renault 2008, cap. 1).

Por tanto, la vía de estudios biográficos que se abrió con la Escuela de Chicago y que se apuntaba en el estudio de Marienthal, que podía haber corregido la desconsideración de Weber, no fue formulada de manera vehemente y quedó clausurada con el estructural-funcionalismo clásico, que paradójicamente se reclamaba heredero de la sociología comprensiva de Weber. Este asunto merece ser explicado con detalle. 


\subsection{Parsons}

En 1930, Talcott Parsons publicó una traducción inglesa de La ética protestante y el espíritu del capitalismo de Weber (Cf. Weber 1930; Parsons 1965), una obra que resulta paradigmática en su uso de la noción weberiana «tipo ideal». En los años siguientes, Parsons intentó integrar las teorías del sociólogo alemán con las aportaciones de Émile Durkheim, Vilfredo Pareto y el economista Alfred Marshall, para buscar una solución original al «problema hobbesiano del orden». Este esfuerzo se plasmó en La estructura de la acción social (libro publicado en 1937), que no ofrece un estudio sociológico, en sentido estricto, sino más bien una formulación sistemática de una teoría de la acción que pretende superar dos dificultades teóricas: la primera, la disolución positivista de la acción voluntaria que, en definitiva, operaba el utilitarismo y su teoría del cálculo interesado (si el individuo se orienta por un mero cálculo, ¿dónde queda su voluntad?); la segunda, la apelación al empirismo con el que resolvía la tradición hobbesiana el problema del orden social, y que dificultaba el establecimiento de un orden normativamente fundamentado, como el que había intentado, por ejemplo, la teoría sociológica de Durkheim. En definitiva, Parsons efectuó una negación lógica del estado de naturaleza: toda sociedad implica un sistema de valores comunes, a partir del cual se seleccionan los objetivos y métodos, y se establecen sus expectativas e interacciones. Parsons apeló a la teoría weberiana de la acción zweckerational, término alemán que fusiona dos expresiones: «dirigida a fines», esto es, teleológica, y «racional». Para centrarnos en el tema de este texto dejaremos de lado el hecho de que ambas expresiones, sobre todo en las tradiciones dialécticas, no resultan exactamente sinónimas. Lo importante es que, focalizando la acción zpeckerational, Parsons desconsideró la primera de las opciones de análisis señalada anteriormente por Weber, la que apuntaba al análisis biográfico. Recuérdese la cita anterior, en la que el sociólogo alemán hablaba de que el análisis del sentido podía considerar «bien a) el sentido efectivo $\alpha$ ) en un caso dado históricamente de una persona actuante, o $\beta$ ) el sentido a modo de promedio y aproximación en una masa dada de casos de las personas actuantes, o b) el sentido supuesto subjetivamente en unos tipos puros construidos conceptualmente...». Pues bien, la definición de acción de Parsons parece olvidar el primer caso (a. $\alpha$ ) y centrarse en el último (b). Así, según el sociólogo norteamericano, la unidad de acción (union act) presenta: i) un «actor» (autor), ii) una finalidad que orienta la acción, iii) una situación, con los medios controlados por el individuo, y iv) una orientación normativa de la acción, sobre la que se realiza el cálculo de medios y fines (una «teleología subjetiva» en expresión de John Elster). El poder regulativo de los valores culturales no altera la «doble contingencia» de la acción, producto de la selección del actor (del ego) y de la selección complementaria del alter. Aunque este elemento fue revisado en los años posteriores, el sentido de la acción siguió interpretándose de manera alejada de las construcciones biográficas. Una descripción sucinta de las etapas del pensamiento de Parsons permite mostrar cómo su sociología se alejó de la posibilidad de abordar estudios biográficos. A partir de los Ensayos en teoría sociológica (obra publicada en 1949), Parsons introdujo «pattern variables of value orientation», patente en su obra Hacia una teoría general de la acción e introdujo la noción de sistema, El sistema social (ambas de 1951), en su estudio de los sistemas analíticos de la acción. El alejamiento de la posibilidad de un estudio biográfico se acentuó a partir de Papeles de trabajo sobre Teoría de Acción (publicados en 1953) y otras obras posteriores, donde se centró en la determinación de variables estructurales. A partir de Teorías de la sociedad (obra de 1961), Parsons elaboró su paradigma de las cuatro funciones (AGIL).

Durante todas las décadas en las que predominó esta orientación estructural-funcionalista, en lugar de los análisis cualitativos de materiales subjetivos y de la importancia de la definición de la situación 
(denominada más tarde por Merton «teorema de Thomas»), tal y como había sido estudiada por la Escuela de Chicago, creció la reputación de los métodos cuantitativos y de una teoría complementaria del sistema social «intacto», que se elaboró de manera diferenciada y definitiva en el funcionalismo estructural de Parsons. El nuevo «armonismo» sociológico, en expresión de Alheit y Dausien, que se postuló en el fondo al precio de la represión intelectual y la rigidez del sistema y de sus subsistemas (cf. Habermas 1973), reprodujo en definitiva la situación de una Norteamérica económicamente fortalecida y en la que, por la Guerra Mundial, se desencadenó un fuerte nacionalismo que arrinconó los problemas de adaptación de los años 20 y 30 del siglo xx.

\section{La corriente crítica}

\section{1. Marx}

Podemos aducir alguna excepción que confirma la regla de que en la filosofía y en la sociología crítica tampoco se cultivaron estudios biográficos. En general, pesó mucho el dictum marxista del prólogo a la Contribución de la crítica de la economía política (1859):

«El modo de producción de la vida material condiciona el proceso vital social, político e intelectual en general. No es la conciencia del ser bumano la que determina su ser, sino que, por el contrario, su ser social es el que determina su conciencia». (MEW XIII: 7-8).

Una afirmación cabalmente repetida por F. Engels o V. I. Lenin, aunque podríamos aducir algunas razones que relativizarían el valor de aquella tesis. La primera es que el texto citado no afirma que el modo de producción determine la biografía. En el pasaje hay dos frases, la primera usa la expresión «condiciona» (bedingt); la segunda, «determina» (bestimmt). Lo que dice el texto es que el modo de producción condiciona el proceso vital (Lebensprozeß), la biografía. Pero este condicionamiento no es óbice para su estudio. La segunda frase afirma que el «ser social» (gesellschaftliches Sein) determina la conciencia, lo que incluye un cierto juego de palabras porque en alemán conciencia (Bewußtsein) incluye la palabra ser (sein); es, literalmente, ser-consciente (bewußt-sein). Que el ser-social determina el ser-consciente podría ser objeto de múltiples interpretaciones y no todas ellas nos alejarían de la investigación biográfica.

La segunda razón por la cual el pasaje citado no tendría que haber conducido a soslayar la investigación biográfica la afirma el propio Marx al principio del prólogo citado, cuando escribe:

"Reprimo una introducción general que habia esbozado, porque con una reflexión más detallada, me parece perturbadora toda anticipación de los resultados a demostrar, y que el lector que quiera seguirme en general, tendrá que ascender de lo particular a lo general. Por el contrario, parecen tener aqui su lugar algunas indicaciones sobre el curso de mis propios estudios político-económicos». (MEW XIII: 7).

Así pues, el pasaje inicial no corresponde a ningún resultado (Resultate) que Marx haya alcanzado y pretenda anticipar, sino que es una de las indicaciones (Andeutungen) que expone para acreditarse como autor que escribe sobre asuntos económicos. El fragmento citado corresponde a la narración del período en el que comenta sus investigaciones en Bruselas con Friedrich Engels, a mediados de los años cuarenta, que dieron origen, entre otros textos, al grueso manuscrito publicado póstumamente como La ideología alemana, donde se encuentran tesis idénticas al pasaje citado y que, dice Marx en el mismo prólogo de 1859, fue abandonado «a la crítica mordaz de los ratones» (MEW XIII: 8). Así pues, no 
se puede deducir que en 1859 Marx siguiera defendiendo aquella tesis. Sin embargo, buena parte del marxismo ha leído El capital, una obra incompleta para su autor, a partir de los textos anteriores, como el Manifiesto del Partido Comunista o La ideología alemana, citada, sin atender a la especificidad de la obra inconclusa.

En El capital, Marx pretendió reproducir la articulación en cuatro planos argumentales de Hegel. Se trata de una re-presentación de lo real que tiene que dar cuenta de una magnitud autoincrementante que, en su despliegue, reproduce las etapas históricas (en el caso de Hegel, la historia universal; en de Marx, la fase más reciente del capitalismo), y que tienen su correlato en la historia de una disciplina destacada (la filosofía y la economía política, respectivamente). Ahora bien, esta representación tiene carácter de análisis formal, previo a las investigaciones empíricas. Así lo afirma explícitamente Marx en algunos pasajes, como el siguiente:

«Aqui solamente tenemos que considerar las formas que atraviesa el capital en sus distintos desarrollos
progresivos. Por tanto, no desarrollaremos las relaciones reales, dentro de las cuales sucede el proceso
real de producción. Supondremos siempre que la mercancia se vende por su valor. No consideraremos
la competencia de los capitales; así como tampoco la banca, ni tampoco la constitución real de la so-
ciedad, que no consiste solamente en las clases de los trabajadores y los capitalistas industriales; y en
la que, por tanto, consumidores y productores no resultan idénticos, ya que la primera categoría, la de
los consumidores (cuyos ingresos son en parte secundarios, ya que derivan del beneficio y del salario, y
no son primarios) es mucho más amplia que la de los segundos, y por ello el modo como los primeros
gastan sus ingresos y el volumen de estos ocasionan modificaciones muy grandes en el presupuesto eco-
nómico, y especialmente en el proceso de circulación y reproducción del capital》. (MEGA 1976: ms.
cuaderno XIII, 704).

Por tanto, Marx pergueñó un modelo incompleto sobre las relaciones sociales, en cuya confrontación empírica no estaba excluida la posibilidad de la indagación biográfica. Sin embargo, tal metodología se dejó de lado, salvo excepciones, precisamente por una interpretación determinista de la conciencia que no se correspondía con lo dicho literalmente por Marx.

\subsection{Kracaver}

A pesar de que la interpretación general marxista soslayó la investigación biográfica, se puede aducir algún caso de estudio relevante dentro de la sociología y la filosofía crítica. Un ejemplo sería la biografía del compositor Offenbach de Siegfried Kracauer (2005). Este, por su formación con Simmel, conoció de primera mano la sociología neokantiana, antes de caer en el círculo de influencia del Instituto de Investigación Social de Fráncfort (cf. Hernàndez 2015).

El libro sobre Offenbach incluye mucho más que una biografía del célebre compositor de operetas. Se mantiene fiel al proyecto de analizar la época mediante sus «discretas expresiones superficiales», para descifrar «el secreto del Segundo Imperio» francés y, en este sentido, cumple con la propuesta de los Pasajes de Benjamin de presentar «imágenes dialécticas» que fueran «cristalizaciones objetivas del movimiento histórico» (Adorno 1995: 22-23). Por ejemplo, cuando Kracauer comenta el primer gran éxito de Offenbach, su obra Orfeo en los infiernos (1858), de la que «fluyen las demás», explica cómo la opereta, «como jugando, ponía al desnudo los fundamentos de la sociedad actual». Y un poco más adelante: «Orfeo desbordó irrefrenablemente el marco de una pieza dramática para convertirse en el emblema de una época» (Kracauer 2005: 184 y 190). En ese sentido, el libro sobre Offenbach cumplía 
con la exigencia planteada en los artículos de cine de Kracauer que luego se reelaboraría en De Caligari a Hitler. Lo «superficial» de las expresiones no solo se refiere a aquello que está en la superficie, sino también a aquella manifestación considerada trivial o menor, en tanto ausente de profundidad, como era el caso de la opereta o del cine. Podríamos pensar en una fenomenología del género menor, del género «chico», como diagnóstico de la época que pasaría por la querella de los bufones (Ferrer 2013), la offenbachiada, la opereta vienesa (Janik \& Toulmin 1998), la zarzuela, los seriales radiofónicos y el cine.

Pero además, en su libro sobre Offenbach, Kracauer apuntaba a un asunto nuclear en el establecimiento de una Teoría Crítica y su concepto de praxis, a saber, la formulación de un mecanismo por el cual no solo se interpreta el orden social vigente, quedando patente, en evidencia, sino que también se explica su transformación. A título de ejemplo, de los muchos que se pueden encontrar en el libro sobre Offenbach, citaré este largo pero notable fragmento:

"Generada en aquel tiempo en que la realidad social era disimulada por la palabra del emperador, [la Offenbachiada] habia intentado ocupar entonces el lugar vacio dejado por esa realidad neutralizada. Ambigua siempre, la opereta habia ejercido bajo la dictadura la función revolucionaria de fustigar el principio de autoridady la corrupción, y trazar bajo falsas apariencies una existencia [Sein] desligada de toda coacción deplorable. Ciertamente, su sátira se disimulaba, a su vez, bajo los velos de la frivolidad; sin duda estaba sofocada por la embriaguez, que respondía a las necesidades del imperio; pero, la frivolidad penetraba más profundamente de lo que bubiese calculado la bohème mundana, ya que la embriaguez, no estaba solo al servicio del aturdimiento y los ataques dirigidos contra el imperio relampagueaban en todo momento. En un tiempo en que la burguesía persistía en abstenerse y en que la izquierda estaba condenada a la impotencia, la opereta de Offenbach era la forma decisiva de la protesta revolucionaria. Su risa taladraba un silencio de encargo y, bajo la apariencia de divertir al público, le incitaba a la oposición. Durante la segunda mitad del imperio se evidenció cada vez. más que era algo diferente a un amable entretenimiento. La Bella Helena despedía peligrosas chispas y La Gran Duquesa apelaba molestamente al emperador.

A medida que se revelaba la irrealidad del Segundo Imperio, resplandecía la realidad de la Offenbachiada. Al mismo tiempo, se tornaba superflua como instrumento político, pues con los retrocesos de la dictadura y los avances de la oposición de izquierda, las fuerzas sociales representadas hasta alli por la Offenbachiada comenzaban a entrar en juego. La capa protectora, al abrigo de la cual habia progresado la Offenbachiada, se bundía poco a poco y la realidad disipaba su lugarteniente, la opereta». (Kracauer 2005: 286-287).

Esta tesis de la opereta como lugarteniente de la realidad, de la existencia social, va más allá, sin duda, del análisis «superficial»; se dirige a lo que podríamos denominar el reconocimiento de los modos de ser sociales, entendiendo «reconocimiento» aquí como algo vinculado a una pugna o lucha, a la manera de la teoría de Axel Honneth. El estudio sobre Offenbach de Kracauer representa, por tanto, un ejemplo notable de cómo una investigación biográfica puede convertirse en un análisis sociológico relevante y no mera narración histórica.

Después del exilio en París, donde escribió la biografía de Offenbach, Kracauer se trasladó a los Estados Unidos. Antes de que arribara, Theodor W. Adorno (1903-1969) ya había sido enrolado como director a tiempo parcial de la sección musical de la Office of Radio Research dirigida por Paul F. Lazarsfeld, con sede en Princeton. Esta iniciativa fracasó y la aportación de fondos de la Fundación Rockefeller fue cancelada en 1939 Picó 2003: 122-123 y 125). Al año siguiente, Lazarsfeld se trasladó 
a Columbia. Más adelante, Adorno participó con otros colaboradores en el estudio de La personalidad autoritaria, en el que, para medir las tendencias antidemocráticas desarrolló, la Escala «F» (por la inicial de «fascismo») (Adorno et al., 1964), «un instrumento de investigación -decía en 1968- que se sigue utilizando continuamente» (Adorno 2006: 35). Este estudio, finalizado en 1950, se insertaba en la serie de Estudios sobre el prejuicio, dirigida por Horkheimer y Samuel H. Flowerman y patrocinado por el American Jewish Committee. Con el tiempo, Adorno expresó la contradicción entre la sociología positivista y la sociología crítica (Adorno 2004: 183-201 y 260-329), y confesó en una de sus últimas clases, a propósito de la metodología cuantitativa, su trabajo con la Escala «F» y su rechazo vehemente del positivismo sociológico: «Soy como el niño que se ha quemado y luego ve el fuego y llora» (Adorno 2006: 35).

Pues bien, si analizamos la trayectoria de Kracauer en Estados Unidos, parece seguir los pasos de aquel Adorno implicado en los estudios empíricos. Kracauer se mantuvo con becas y ayudas de diversas fundaciones (Fundación Rockefeller, Fundación Bollingen, Fundación Chapelbrook de Boston) (Traverso 1998: 176) hasta ocupar el puesto de Director of Research for Applied Social Sciences en la Universidad de Columbia. Por el camino, había firmado con Leo Löwenthal (1900-1993, antes del exilio, colaborador de Horkheimer en el Instituto de Investigación Social de Fráncfort d. M.) y Lazarsfeld la investigación Satellite Mentality (realizada en 1956), en el que se analizaban 300 entrevistas a emigrados que procedían de Hungría, Polonia y Checoslovaquia, entonces bajo dominio del gobierno soviético (Traverso 1998: 198), un trabajo que parece seguir el sometimiento de la investigación auspiciada por las fundaciones mencionadas a los intereses propagandísticos del complejo militar industrial estadounidense (Picó 2003, 81-102). Con todo, la aportación más célebre de Kracauer fueron sus estudios sobre el cine, que exceden el ámbito de este texto.

\section{Conclusión}

Como hemos mostrado, en las principales corrientes sociológicas no había un impedimento teórico relevante para no desarrollar investigaciones biográficas. Sin embargo, una determinada lectura de Parsons de las propuestas metodológicas de Weber, que a su vez se habían orientado, de manera cuestionable, y una interpretación de los escritos de Marx, en contra de sus propios textos, alejaron tanto a las corrientes comprensivas como a las críticas de la investigación biográfica.

\section{Referencias bibliográficas}

Adorno, Theodor W. et al., (1964). The Authoritarian Personality, 2 vols. Nueva York: John Wiley \& Sons.

Adorno, Theodor W. (1995). Sobre Walter Benjamin. Madrid: Cátedra.

Adorno, Theodor W. (2004). Escritos sociológicos I (Obra completa, 8). Madrid: Akal.

Adorno, Theodor W. (2006). Introducción a la sociología. Barcelona: Gedisa.

Alheit, Peter \& Dausien, Bettina (2009): “'Biographie' in den Sozialwissenschaften” en Bernhard Fetz (ed.): Die Biographie. Zur Grundlegung ihrer Theorie. Berlín, Nueva York: Walter de Gruyter.

Ferrer, Anacleto (2013). La Querella de los Bufones. València: Publicacions de la Universitat de València. Foucault, Michel (1981). Vigilary castigar. Nacimiento de la prisión. Madrid: Siglo XXI. 
Habermas, Jürgen (1973): "Stichworte zur Theorie der Sozialisation" [1968] en Id.: Kultur und Kritik. Fráncfort d. M.: Suhrkamp.

Hernàndez, Francesc Jesús (2015): “Kracauer y la Escuela de Frankfurt” en Susana Díaz (ed.): Historia y teoría crítica. Lectura de Siegfried Kracauer. Madrid: Biblioteca Nueva.

Janik, Allan \& Toulmin, Stephen (1998). La Viena de Wittgenstein. Madrid: Taurus.

Kracauer, Siegfried (2005). Jacques Offenbach und das Paris seiner Zeit (Werke VIII). Fráncfort d. M.: Suhrkamp.

Marx, Karl (MEGA) (1976). Marx-Engels Gesamtausgabe. Berlín: Akademie, vol. II. 3, pp. 1113-1155.

Marx, Karl (MEW) (1961). Marx-Engels Werke. Berlín: Dietz Verlag, vol. XIII.

Parsons, Talcott (1965): "Evaluation et objectivité dans le domaine des sciences sociales: une interprétation des travaux de Max Weber”. Revue Internationale des Sciences Sociales, vol. XVII, núm. 1, 49-69.

Picó, Joseph (2003). Los años dorados de la sociología (1945-1975). Madrid: Alianza.

Poca, Anna (1991): "Estudio introductorio" en F. Schleiermacher: Monólogos, Barcelona: Anthropos; Madrid: Ministerio de Educación y Ciencia.

Renault, Emmanuel (2008). Souffrances sociales. Sociologie, psychologie et politique. París: La Découverte.

Schleiermacher, Friedrich Daniel Ernst (1868). Monologen, Berlín (transcripción en http://zeno.org).

Schleiermacher, Friedrich Daniel Ernst (1985). Über die Religion. Stuttgart: Reclam.

Thomas, William Isaac \& Znaniecky, Florian (1918-1920). The Polish Peasant in Europa and America, Boston: Gorham Press.

Traverso, Enzo (1998). Siegfried Kracauer. Itinerario de un intelectual nómada. Valencia: Alfons el Magnànim.

Weber, Marianne (1995). Max Weber. Una biografía. València: Institució Alfons el Magnànim.

Weber, Max (1930). The Protestant Ethic and the Spirit of Capitalism, Nueva York: Scribner's. Trad. Talcott Parsons

Weber, Max (1973). Ensayos sobre metodología sociológica. Buenos Aires: Amorrortu.

Weber, Max (1980): Wirtschaft und Gesellschaft. Grundriß der verstehenden Soziologie [1921-1922], 5a ed. revisada de Johannes Winckelmann. Studienausgabe, Tubinga: J. C. B. Mohr (Paul Siebeck).

Znaniecki, Florian (1969). On Humanistic Sociology. Selected Papers, edic. Robert Bierstedt, Chicago.

\section{Nota biográfica}

María Eugenia Cardenal de la Nuez es profesora de sociología de la educación. Ha desarrollado investigaciones biográficas sobre transiciones formativas. Ha sido coeditora del libro Memory, Subjectivities, and Representation Approaches to Oral History in Latin America, Portugal, and Spain. Nueva York: Palgrave Macmillan.

Francesc J. Hernàndez es profesor de sociología de la educación de la Universitat de València. Ha publicado, junto con Alícia Villar, Biografías y educación. Barcelona: UOC, 2015, y diversas traducciones de Peter Alheit y Bettina Dausien. 